\title{
The pulsar population in Globular Clusters and in the Galaxy
}

\author{
Paulo C. C. Freire \\ Max-Planck-Institut für Radioastronomie, auf dem Hügel 69, 53121, Bonn, Germany \\ email: pfreire@mpifr-bonn.mpg.de
}

\begin{abstract}
In this paper, I review some of the basic properties of the pulsar population in globular clusters (GCs) and compare it with the the Galactic disk population. The neutron stars (NSs) in GCs were likely formed - and appear to continue forming - in highly symmetric supernovae (SNe), likely from accretion-induced collapse (AIC). I review the many pulsar finds and discuss some particularly well populated GCs and why they are so. I then discuss some particularly interesting objects, like millisecond pulsars (MSPs) with eccentric orbits, which were heavily perturbed by passing stars. Some of these systems, like NGC 1851A and NGC 6544B, are almost certainly the result of exchange interactions, i.e., they are witnesses to the very same processes that created the large population of MSPs in the first place. I also review briefly the problem posed by the presence of young pulsars in GCs (with a special emphasis on a sub-class of young pulsars, the super-energetic MSPs), which suggest continuing formation of NSs in low-velocity SNe. In the final section, I discuss the possibility of an analogous population in the Galaxy and highlight a particularly interesting case, PSR J1903+0327, where the primary neutron star appears to have formed with a small-velocity kick and small fractional mass loss. Systems with primary NSs formed in electron-capture SNe should constitute a distinct low-velocity Galactic population akin in many respects to the GC population. Current high-resolution surveys of the Galactic plane should be able to detect it clearly.
\end{abstract}

Keywords. (Galaxy:) globular clusters: general, stars: neutron, (stars:) pulsars: general, X-rays: binaries, (stars:) binaries: eclipsing

\section{The Pulsar population in Globular Clusters}

Globular clusters (GCs) are spherical, bound swarms of stars containing from $10^{4}$ to $\sim 5 \times 10^{6}$ stars. Near their centers the star density is normally over $10^{3}$ (and in some cases $10^{6}$ !) per cubic parsec. They orbit the centers of most Galaxies through the Universe, about 200 orbit our Milky Way (Harris 1996). Of these, 28 clusters contain a total of 144 known radio pulsarst.

The GC pulsar population differs from the Galactic disk population in two main ways:

(a) It is much older than the Galactic population. This is to be expected given the great age of the stellar population in GCs. This population is so old that, with a few important exceptions (discussed below), only recycled pulsars, which have lifetimes of many Gyr, are still detectable as radio pulsars.

(b) It is a very abundant population. Per unit mass there appear to be two to three orders of magnitude more pulsars in GCs as in the Galactic disk. This also applies to X-ray sources, the progenitors of MSPs.

The reasons for this latter fact, and its many consequences, are discussed in detail below.

$\dagger$ Our updated reference list is at http://www.naic.edu/ pfreire/GCpsr.html 


\section{Large neutron star population in globular clusters}

\subsection{Exchange encounters}

In the early 1970's, the Uhuru and OSO-7 X-ray satellites revealed the presence of several X-ray sources in GCs (e.g. Giacconi et al. 1974). One of the co-authors (H. Gursky) recognized in 1973 that, compared to the stellar mass in the Galaxy, this represented a large overabundance of X-ray sources. In 1975 G. Clark suggested that, given the extremely high stellar densities in the cores of some GCs, it occasionally happens that many old, dead NSs lurking in the core "collide" with a binary, disrupt it and acquire a (new) companion. The latter then evolves, fills its Roche lobe and starts transferring matter to the NS, forming a low-mass X-ray binary (LMXB).

\subsection{Origin of large NS population}

An important question already considered in these early studies is the origin of all these lurking NSs. In the early 1970's some pulsar proper motions had already been measured (e.g., Manchester et al. 1974) and these already hinted at the fact that many NSs form with kick velocities of hundreds of $\mathrm{km}$ per second. This has since been confirmed by many subsequent studies, with ever-increasing samples and better quality measurements (Lyne \& Lorimer 1994, Hobbs et al. 2005). Such objects would not be retained in GCs, which have escape velocities of a few tens of $\mathrm{km} \mathrm{s}^{-1}$ — unless they are anchored by massive companions (Davies \& Hansen 1998). Even with such anchoring, there seems to be a large excess of NSs (Pfahl, Rappaport \& Podsiadlowski 2002), which lead to a suggestion, still valid (originally by Katz 1975) that NSs in GCs are forming through a low-velocity channel. This is now thought (Podsiadlowski et al. 2004) to be accretion-induced collapse (AIC) of massive Oxygen-Neon-Magnesium WDs that become unstable once they approach the Chandrasekhar limit. This results in electron capture SNe (Poelarends et al. 2008, Langer 2012), not Type 1a SNe - there is not enough carbon available to power thermonuclear deflagrations. We will come back to this issue later.

\section{Millisecond pulsars - in the Galaxy and in GCs}

Soon after the discovery of the first MSP, B1937+21 in 1982 (Backer et al. 1982), it was suggested that MSPs are the end stages of the evolution of LMXBs (Alpar et al. 1982). This is consistent with the finding that, unlike in the case of normal pulsars, most MSPs are found in binary systems. Here the mystery is why some of these objects (including B1937+21 itself) are found to be isolated; there is no satisfactory answer to this question yet. Furthermore, MSPs have magnetic fields much smaller than those generally found in the normal pulsar population; which means that accretion somehow "buries" the magnetic field. This process is not well understood.

If MSPs really evolve from LMXBs, then GCs, with their LMXB over-abundance, should also contain many MSPs. Finding them was difficult, given the great distances to GCs. Nevertheless, if a bright radio source is discovered in a GC, then it is likely a pulsar. In 1985, twelve nearby GCs were imaged with the Very Large Array (VLA, Hamilton, Helfand \& Becker 1985), with one likely candidate in M28, B1821-24. Two years later, using the Jodrell Bank 75 -m radio telescope, the discovery of radio pulsations at a period of $3.05 \mathrm{~ms}$ and DM of $120 \mathrm{~cm}^{-3} \mathrm{pc}$ confirmed it as the first pulsar in a GC (Lyne et al. 1987). In the following year, PSR B1620-26 is discovered in the globular cluster M4 (Lyne et al. 1988). This pulsar, with a spin period of $11 \mathrm{~ms}$, is in a 191-day orbit with a white dwarf (WD). Interestingly, this binary system appears to be orbited by a Jovian-type planet in a very wide orbit (Sigurdsson et al. 2003). 


\subsection{Finding more}

The pulsars in GCs are faint owing to their great distances. This means that we are limited first and foremost by the sensitivity of the radio telescope and receiver used for the survey. This can be, to some extent, compensated by the fact that the pulsars are located in a small region around the cluster center (owing to the effects of mass segregation, see e.g, Freire et al. 2001a) which normally fits very well inside a single radio beam of even the largest radio telescopes. This allows for deep, multi-hour search observations, which can still be made sensitive to binary pulsars using acceleration search techniques (Camilo et al. 2000, Ransom et al. 2002). This is the reason why for most of the last 20 years we knew more MSPs in clusters than in the Galactic disk.

\subsection{Notable clusters and why they are so}

The early leader in pulsar discoveries was 47 Tucanae (Manchester et al. 1990, Manchester et al. 1991, Robinson et al. 1995, Camilo et al. 2000), which now has a total of 23 known pulsars, all of them with spin periods shorter than $7.6 \mathrm{~ms}$. Of these, 15 are in binary systems, at least five of them eclipsing. The timing of these pulsars (Freire et al. 2001a) allowed X-ray detections of all of the MSPs (Heinke et al. 2005, Bogdanov et al. 2006), a study of the dynamics of the cluster (Freire et al. 2003) and the first detection of any sort of interstellar medium in a globular cluster, after more than 60 years of searches (Freire et al. 2001b).

However, radio maps of a large group of GCs (Fruchter \& Goss 2000) suggested that the heavily obscured GC Terzan 5 had a large pulsar population, but of these only two were known before that study (Lyne et al. 1990, Lyne et al. 2000). Sensitive observations at 2 GHz with the GBT have since discovered 32 pulsars (Ransom et al. 2005, Hessels et al. 2006). These include Terzan 5 ad (Hessels et al. 2006): with a spin frequency of $716 \mathrm{~Hz}$; this broke the 24-year old record set by the original MSP, B1937+21. It is thought that $\sim 100$ pulsars remain to be discovered in Terzan 5 (Bagchi, Lorimer \& Chennamangalam 2011).

Using the same observing system 8 new pulsars were found in NGC 6440 and NGC 6441 (Freire et al. 2008a) and 11 new pulsars in M28 alone! Freire et al. (2008a) found that, for any particular luminosity threshold, there are nearly as many pulsars in NGC 6440 and NGC 6441 as there are in Terzan 5. The latter appears to be exceptional because of its smaller distance to the Solar System $(5.5 \mathrm{kpc})$, as opposed to $8.2 \mathrm{kpc}$ for NGC 6440 and $13.5 \mathrm{kpc}$ for NGC 6441 . This results highlights the fact that these surveys are strongly limited by sensitivity.

It is nevertheless clear that, after correcting for distance, some GCs have many more pulsars than the average. Although we don't know all the factors involved in producing a large pulsar population, the stellar encounter rate has some predictive power not only in estimating the number of MSPs, but of other types of objects (e.g., Davies 1995), particularly X-ray binaries (e.g., Pooley et al. 2003).

\subsection{Clusters with isolated pulsars}

The pulsar populations of some clusters [NGC 7078 (Anderson 1993), NGC 6624 (Biggs et al. 1994, Lynch et al. 2012), NGC 6517 (Lynch et al. 2011) and NGC 6752 (D'Amico et al. 2001, D'Amico et al. 2002)] are dominated by isolated pulsars, while others [like 47 Tuc (Camilo et al. 2000), M62 (Possenti et al. 2003, Lynch et al. 2012), M3, M5 and M13 (Kulkarni et al. 1991, Anderson et al. 1997, Hessels et al. 2007)] are dominated by binaries. The distinguishing characteristic appears to be the core density; most of the GCs where there are more isolated pulsars appear to be core collapsed. The high stellar density at the core might be disrupting previously formed binary MSPs. Another possible 
disrupter are central black hole binaries, as has been suggested in the case of NGC 6752 (Colpi, Possenti \& Gualandris 2002).

Furthermore, many of the GCs dominated by isolated pulsars appear to have a slower pulsar population (Verbunt 2003), even in surveys where such clusters are observed with uniform sensitivity to fast-spinning pulsars, as in the comparison of M3/M5/M13 with M15 (Hessels et al. 2007) or NGC 6440/6441 with Terzan 5 (Freire et al. 2008a). It is likely that the high stellar densities of some GC cores are also disrupting X-ray binaries, leaving behind partially recycled pulsars.

\subsection{Young pulsars in globular clusters}

Some pulsars in GCs [PSR B1718-19 in NGC 6342 (Lyne et al.1993), PSR B1820-30B (Biggs et al. 1994) and J1823-3021C (Lynch et al. 2012) in NGC 6624 and B1745-20 in NGC 6440 (Lyne et al. 1996)] have characteristics very similar to the normal pulsars found in the Galactic disk: periods of the order of a few tenths of a second, magnetic fields of the order of $10^{11-12} \mathrm{G}$ and characteristic ages of a few times $10^{7} \mathrm{yr}$ - about $10^{3}$ times younger than the stellar population in GCs. Clearly these pulsars cannot have formed in recent iron core collapse $\mathrm{SNe}$ - there have not been any since the first few tens of Myr of the histories of these clusters. The partial recycling described above could be spinning up old, dead NSs just enough to make them active radio pulsars, but without going on for long enough to bury their magnetic fields (Lyne et al. 1996).

An alternative hypothesis, also discussed in Lyne et al. (1996) is ongoing formation of new NSs through e-capture SNe. If this hypothesis is correct, then the SNe must have small kicks, otherwise, the formation rates required by the observed population would be unrealistic (Boyles et al. 2011).

\section{Exotic pulsars in GCs}

\subsection{Eccentric binary MSPs $\&$ their uses}

The first pulsar in an eccentric binary discovered in a GC was PSR B2127+11C (Anderson et al. 1990). This is very similar to the original binary pulsar, B1913+16 (Hulse \& Taylor 1975, Weisberg et al. 2010), so it does not indicate anything special is happening in GCs. The discovery of PSR B1802-07, in the GC NGC 6539 (D'Amico et al. 1993) revealed a type of system unknown in the Galactic disk: a relatively fast-spinning pulsar $(P=23.1 \mathrm{~ms})$ with a low-mass $\left(\mathrm{M}_{\mathrm{c}} \sim 0.3 \mathrm{M}_{\odot}\right)$ companion an an eccentric $(e=0.21)$ orbit. All similar systems then known in the Galaxy had very low $\left(<10^{-3}\right)$ eccentricities. This indicated severe orbital perturbations by passing stars (Phinney 1993), something to be expected given the high stellar densities in the cores of GCs.

The discovery of PSR J0514-4002A, in NGC 1851, an MSP with a spin period of $4.99 \mathrm{~ms}$, a very eccentric $(e=0.888)$ 18.8-day orbit (Freire et al. 2004) and massive companion indicates very conclusively that the pulsar exchanged companions after being recycled: the companion is too massive $\left(\mathrm{M}_{\mathrm{c}}>0.96 \mathrm{M}_{\odot}\right.$, Freire, Ransom \& Gupta 2007) for its progenitor to have recycled the pulsar to its current spin period. This is also the case for at least another system, PSR J1807-2500B, in NGC 6544 (Lynch et al. 2012). Thus, this sort of system bears witness to the very same process that lead to the recycling of so many pulsars in GCs. Their dense environments can produce binary pulsars (and binary systems in general) that are truly unlike anything that binary stellar evolution can produce in the Galactic disk. These are the systems we designate here as "exotic".

A total of 19 eccentric $(e>0.2)$ systems have been discovered in GCs (e.g. Ransom et al. 2004, Possenti et al. 2005, Freire et al. 2008a, DeCesar, Ransom \& Ray 2011, Lynch et al. 2012), including 7 such systems in Terzan 5 alone (Ransom et al. 2005). 
The eccentricities allow at least the measurement of the rate of advance of periastron. When this effect is due to the effects of general relativity alone it yields an estimate of the total mass of the binary (D'Amico et al. 1993, Freire et al. 2003, Ransom et al. 2005, Freire, Ransom \& Gupta 2007, Freire et al. 2008a, Freire et al. 2008b). When more Post-Keplerian measurements become available, we can measure individual masses precisely and in some cases test general relativity (Lynch et al. 2012, Jacoby et al. 2006).

\subsection{Eclipsing binaries}

Until recently, it appeared that one of the distinctive characteristics of the pulsar population in GCs was the large number of eclipsing binaries: 21 known at present. Until 2009, only two were known in the Galactic disk, the original "Black Widow" system, PSR B1957+20 (Fruchter, Stinebring \& Taylor) and PSR J2051-0827 (Stappers et al. 1996). Furthermore, several of the eclipsing systems discovered in GCs [e.g., PSR B171819 in NGC 6342 (Lyne et al.1993), PSR J1748-2446A, P and ad in Terzan 5 (Lyne et al. 1990, Ransom et al. 2005, Hessels et al. 2006), J0024-7204W in 47 Tuc (Camilo et al. 2000, Edmonds et al. 2002), J1740-5340 in NGC 6397 (D'Amico et al. 2001, D'Amico et al. 2001, Ferraro et al. 2001), J1701-3006B in M62 (Possenti et al. 2003) and PSR J2140-2310A in M30 (Ransom et al. 2004)] had in some cases very extensive eclipses and non-degenerate companions with a few tenths of a solar mass. Because these systems (nicknamed "Redbacks" by Mallory Roberts) had no counterpart in the Galaxy, they were thought to be "exotic", i.e., results of exchange interactions where a radio pulsar acquires a new main-sequence companion.

However, it was suggested that, due to its similarity with SAX J1808.4-3658 (the first accreting MSP, Wijnands \& van der Klis 1998), 47 Tuc W might also represent a transitional object (Bogdanov et al. 2005), not an exotic system. The building of the LMXB-MSP bridge continued with the discovery of PSR J1023+0038 (Archibald et al. 2009), the first Galactic "Redback", which showed these objects are not restricted to GCs. The number of eclipsing systems in the Galaxy has since increased dramatically, in great part due to the launch of the Fermi satellite. The tale is told by Mallory Roberts in these proceedings. The "Black Widow" and "Redback" systems are much more abundant in the Galactic disk than previously thought. Despite this, some eclipsing systems in GCs are likely to be truly "exotic", like PSR B1718-19 (van Kerkwijk et al. 2000).

\subsection{Super-energetic MSPs: in GCs and in the Galaxy?}

In GCs, there are two MSPs that appear to have unusually high magnetic fields, very large spin-down energies and ages smaller than $30 \mathrm{Myr}$ - about $0.3 \%$ of the age of the clusters that host them. One of them is the first GC pulsar, PSR B1821-24. Soon after its period derivative $(\dot{P})$ was measured (Foster et al. 1988), the unusual nature of the MSP was noticed and discussed. It was deemed to be unlikely that a contribution from the cluster acceleration could be the cause for the anomalous $\dot{P}$.

There is firmer evidence of this for the second "super-energetic" MSP to be discovered, PSR B1820-30A in NGC 6624 (Biggs et al. 1994). Initially the discoverers suggested that the very high $\dot{P}$ was due to acceleration in the cluster; this is a possibility given that the cluster has a collapsed core and the pulsar is (at least in projection) very close to the center. However, the high $\gamma$-ray luminosity of this object (Freire et al. 2011) implies that this pulsar has to be energetic and quite young.

These MSPs have lifetimes $\sim 10^{2}$ times shorter than the more normal MSPs and are about $10^{2}$ times less abundant; therefore both types must be forming at comparable rates. We list three main possibilities for their formation process: (a) These pulsars were members of now disrupted X-ray binaries, where spin-up went much further than for the 
young, slow pulsars, but where the "burial" of the magnetic field was not concluded. This would nicely explain why both objects are single, (b) These pulsars could result from AIC or merger-induced collapse of WDs (Ivanova et al. 2008), (c) They might have an identical formation channel to other MSPs (Tauris, Langer \& Kramer 2012). If (a) is correct, then these super-energetic MSPs are "exotic", i.e., only found in GCs. Otherwise we should see similar systems in the Galaxy, likely associated with gamma-ray sources. As the present generation of high-resolution 20-cm surveys probes deeper into the Galaxy for MSPs (Cordes et al. 2006, Keith et al. 2010, Boyles et al. 2012, Lynch et al. 2012) we might soon know whether pulsars like PSR B1820-30A and B1821-24A exist outside GCs or not. Finding them would have grand implications: it would mean that they are forming at rates similar to normal MSPs through the Universe and that MSPs are born with a range of magnetic fields wider than currently believed; the observation that the majority of MSPs have very low B-fields would then be a selection effect caused by low B-field MSPs being much longer lived.

\section{Is there a low-velocity NS population in the Galaxy?}

If e-capture SNe are forming NSs in GCs, they should also be forming NSs in the Galaxy: nothing about them is exclusive to GCs. A low-velocity NS population has indeed been suggested several times from proper motion data of radio pulsars (e.g., Arzoumanian, Chernoff \& Cordes 2002), but this signature is not clear and could be due instead to projection effects (Hobbs et al. 2005). This might be explained if e-capture SNe result only from AIC of a massive O-Ne-Mg WD (or, alternatively, from the merger of two WDs), i.e., if it requires evolution in a binary system (Podsiadlowski et al. 2004). If this is true, then binary systems are the place to look for evidence of NSs formed in e-capture SNe in the Galaxy.

The binaries seem to agree. Pfahl et al. (2002) identified a class of high-mass, long orbital period $\left(P_{b}>30\right.$ days) low-eccentricity X-ray binaries (the prime example being $\mathrm{X}$ Per/4U 0352+309), in which the NSs must have been born with a low kick velocity. Later, remarking the low eccentricities of several double neutron star systems, van den Heuvel (2004) suggested that the second-born NSs in those systems were produced by e-capture SNe. An additional piece of evidence in these systems is the NS mass measurements, some of them as low as $1.25 \mathrm{M}_{\odot}$ (e.g., Kramer et al. 2006), as expected from the gravitational collapse of a contracting O-Ne-Mg core that is just beyond its Chandrasekhar mass $\left(1.38 \mathrm{M}_{\odot}\right.$, see Schwab, Podsiadlowski \& Rappaport 2010 and references therein).

There is also some evidence for systems where the first formed NS has a very low mass and low velocity, (PSR J1802-2124; Ferdman et al. 2010), or a very small vertical velocity and possibly a small NS mass (PSR J1949+3106; Deneva et al. 2012), however the massive WD progenitors should have diminished post-SN velocities of these binaries. Another case is PSR J1903+0327 (Champion et al. 2008), the first MSP discovered in the ALFA pulsar survey (Cordes et al. 2006). This anomalous pulsar was likely formed in a triple system (Freire et al. 2011, Portegies Zwart et al. 2011), but the preservation of the triple requires a very small kick velocity (Pijloo, Caputo \& Portegies Zwart 2012); this is consistent with the very small peculiar velocity of this system (Freire et al. 2011).

Systems where the first NS formed in e-capture SNe should constitute a dynamically separate NS population in the Galaxy with very low scale height; furthermore many of the NSs should have distinctively low masses. Proper motion measurements of the many MSPs being discovered in the current high-resolution $20 \mathrm{~cm}$ surveys should be able to determine whether such a population exists and determine its size. 


\section{Acknowledgments}

I thank the financial support by the European Research Council for the ERC Starting Grant BEACON under contract no. 279702.

\section{References}

Alpar, M. A., Cheng, A. F., Ruderman, M. A., \& Shaham, J. 1982, Nature, 300, 728

Anderson, S. B. 1993, Ph.D. Thesis, Caltech

Anderson, S. B., Gorham, P. W., Kulkarni, S. R., Prince, T. A., \& Wolszczan, A. 1990, Nature, 346,42

Anderson, S. B., Wolszczan, A., Kulkarni, S. R., \& Prince, T. A. 1997, Apj, 482, 870

Archibald, A. M., Stairs, I. H., Ransom, S. M., et al. 2009, Science, 324, 1411

Arzoumanian, Z., Chernoff, D. F., \& Cordes, J. M. 2002, ApJ, 568, 289

Backer, D. C., Kulkarni, S. R., Heiles, C., Davis, M. M., \& Goss, W. M. 1982, Nature, 300, 615 Bagchi, M., Lorimer, D. R., \& Chennamangalam, J. 2011, MNRAS, 418, 477

Biggs, J. D., Bailes, M., Lyne, A. G., Goss, W. M., \& Fruchter, A. S. 1994, MNRAS, 267, 125

Bogdanov, S., Grindlay, J. E., \& van den Berg, M. 2005, ApJ, 630, 1029

Bogdanov, S., Grindlay, J. E., Heinke, C. O., et al. 2006, ApJ, 646, 1104

Boyles, J., Lorimer, D. R., Turk, P. J., et al. 2011, ApJ, 742, 51

Boyles, J., Lynch, R., Ransom, S. M., et al. 2012, arXiv:1209.4293

Camilo, F., Lorimer, D. R., Freire, P., Lyne, A. G., \& Manchester, R. N. 2000, ApJ, 535, 975

Champion, D. J., Ransom, S. M., Lazarus, P., et al. 2008, Science, 320, 1309

Clark, G. W. 1975, ApJ, 199, L143

Colpi, M., Possenti, A., \& Gualandris, A. 2002, ApJ, 570, L85

Cordes, J. M., Freire, P. C. C., Lorimer, D. R., et al. 2006, ApJ, 637, 446

D'Amico, N., Bailes, M., Lyne, A. G., et al. 1993, MNRAS, 260, L7

D'Amico, N., Lyne, A. G., Manchester, R. N., Possenti, A., \& Camilo, F. 2001, ApJ, 548, L171

D'Amico, N., Possenti, A., Manchester, R. N., et al. 2001, ApJ, 561, L89

D'Amico, N., Possenti, A., Fici, L., et al. 2002, ApJ, 570, L89

Davies, M. B. 1995, MNRAS, 276, 887

Davies, M. B. \& Hansen, B. M. S. 1998, MNRAS, 301, 15

DeCesar, M. E., Ransom, S. M., \& Ray, P. S. 2011, arXiv:1111.0365

Deneva, J. S., Freire, P. C. C., Cordes, J. M., et al. 2012, ApJ, 757, 89

Edmonds, P. D. et al. 2002, ApJ, 579, 741

Ferdman, R. D., Stairs, I. H., Kramer, M., et al. 2010, ApJ, 711, 764

Ferraro, F. R., Possenti, A., D'Amico, N., \& Sabbi, E. 2001, ApJ, 561, L93

Foster, R. S., Backer, D. C., Taylor, J. H., \& Goss, W. M. 1988, ApJ, 326, L13

Freire, P. C. C., Abdo, A. A., Ajello, M., et al. 2011, Science, 334, 1107

Freire, P. C. C., Bassa, C. G., Wex, N., et al. 2011, MNRAS, 412, 2763

Freire, P. C., Camilo, F., Lorimer, D. R., et al. 2001, MNRAS, 326, 901

Freire, P. C., Camilo, F., Kramer, M., et al. 2003, MNRAS, 340, 1359

Freire, P. C., Gupta, Y., Ransom, S. M., \& Ishwara-Chandra, C. H. 2004, ApJ, 606, L53

Freire, P. C., Kramer, M., Lyne, A. G., et al. 2001, ApJ, 557, L105

Freire, P. C. C., Ransom, S. M., Bégin, S., et al. 2008, ApJ, 675, 670

Freire, P. C. C., Ransom, S. M., \& Gupta, Y. 2007, ApJ, 662, 1177

Freire, P. C. C., Wolszczan, A., van den Berg, M., \& Hessels, J. W. T. 2008, ApJ, 679, 1433

Fruchter, A. S. \& Goss, W. M. 2000, ApJ, 536, 865

Fruchter, A. S., Stinebring, D. R., \& Taylor, J. H. 1988, Nature, 333, 237

Giacconi, R., Murray, S., Gursky, H., et al. 1974, ApJ. Supp., 27, 37

Hamilton, T. T., Helfand, D. J., \& Becker, R. H. 1985, AJ, 90, 606

Harris, W. E. 1996, AJ, 112, 1487 (http://physwww.mcmaster.ca/ harris/mwgc.dat).

Heinke, C. O., Grindlay, J. E., Edmonds, P. D., et al. 2005, ApJ, 625, 796

van den Heuvel, E. P. J. 2004, 5th INTEGRAL Workshop on the INTEGRAL Universe, 552, 185 
Hessels, J. W. T., Ransom, S. M., Stairs, I. H., et al. 2006, Science, 311, 1901

Hessels, J. W. T., Ransom, S. M., Stairs, I. H., Kaspi, V. M., \& Freire, P. C. C. 2007, ApJ, 670, 363

Hobbs, G., Lorimer, D. R., Lyne, A. G., \& Kramer, M. 2005, MNRAS, 360, 974

Hulse, R. A. \& Taylor, J. H. 1975, ApJ, 195, L51

Ivanova, N., Heinke, C. O., Rasio, F. A., Belczynski, K., \& Fregeau, J. M. 2008, MNRAS, 386, 553

Jacoby, B. A., Cameron, P. B., Jenet, F. A., et al. 2006, ApJ, 644, L113

Katz, J. I. 1975, Nature, 253, 698

Keith, M. J., Jameson, A., van Straten, W., et al. 2010, MNRAS, 409, 619

Kramer, M., Stairs, I. H., Manchester, R. N., et al. 2006, Science, 314, 97

van Kerkwijk, M. H., Kaspi, V. M., Klemola, A. R., et al. 2000, ApJ, 529, 428

Kulkarni, S. R., Anderson, S. B., Prince, T. A., \& Wolszczan, A. 1991, Nature, 349, 47

Langer, N. 2012, Annual Review of Astronomy and Astrophysics, 50, 107

Lynch, R. S., Boyles, J., Ransom, S. M., et al. 2012, arXiv:1209.4296

Lynch, R. S., Freire, P. C. C., Ransom, S. M., \& Jacoby, B. A. 2012, ApJ, 745, 109

Lynch, R. S., Ransom, S. M., Freire, P. C. C., \& Stairs, I. H. 2011, ApJ, 734, 89

Lyne, A. G., Biggs, J. D., Brinklow, A., McKenna, J., \& Ashworth, M. 1988, Nature, 332, 45

Lyne, A. G., Biggs, J. D., Harrison, P. A., \& Bailes, M. 1993, Nature, 361, 47

Lyne, A. G., Brinklow, A., Middleditch, J., Kulkarni, S. R., \& Backer, D. C. 1987, Nature, 328, 399

Lyne, A. G., Johnston, S., Manchester, R. N., Staveley-Smith, L., \& D’Amico, N. 1990, Nature, 347,650

Lyne, A. G. \& Lorimer, D. R. 1994, Nature, 369, 127

Lyne, A. G., Manchester, R. N., \& D'Amico, N. 1996, ApJ, 460, L41

Lyne, A. G., Mankelow, S. H., Bell, J. F., \& Manchester, R. N. 2000, MNRAS, 316, 491

Manchester, R. N., Lyne, A. G., Johnston, S., et al. 1990, Nature, 345, 598

Manchester, R. N., Lyne, A. G., Robinson, C., Bailes, M., \& D’Amico, N. 1991, Nature, 352, 219

Manchester, R. N., Taylor, J. H., \& Van, Y. Y. 1974, ApJ, 189, L119

Pfahl, E., Rappaport, S., \& Podsiadlowski, P. 2002, ApJ, 573, 283

Pfahl, E., Rappaport, S., Podsiadlowski, P., \& Spruit, H. 2002, ApJ, 574, 364

Phinney, E. S. 1993, Structure and Dynamics of Globular Clusters, 50, 141

Pijloo, J. T., Caputo, D. P., \& Portegies Zwart, S. F. 2012, MNRAS, 424, 2914

Podsiadlowski, P., Langer, N., Poelarends, A. J. T., et al. 2004, ApJ, 612, 1044

Poelarends, A. J. T., Herwig, F., Langer, N., \& Heger, A. 2008, ApJ, 675, 614

Pooley, D., Lewin, W. H. G., Anderson, S. F., et al. 2003, ApJ, 591, L131

Portegies Zwart, S., van den Heuvel, E. P. J., van Leeuwen, J., \& Nelemans, G. 2011, ApJ, 734, 55

Possenti, A., D’Amico, N., Corongiu, A., et al. 2005, Binary Radio Pulsars: AST Conference Series 328,189

Possenti, A., D'Amico, N., Manchester, R. N., et al. 2003, ApJ, 599, 475

Ransom, S. M., Eikenberry, S. S., \& Middleditch, J. 2002, AJ, 124, 1788

Ransom, S. M., Hessels, J. W. T., Stairs, I. H., et al. 2005, Science, 307, 892

Ransom, S. M., Stairs, I. H., Backer, D. C., et al. 2004, ApJ, 604, 328

Robinson, C., Lyne, A. G., Manchester, R. N., et al. 1995, MNRAS, 274, 547

Schwab, J., Podsiadlowski, P., \& Rappaport, S. 2010, ApJ, 719, 722

Sigurdsson, S., Richer, H. B., Hansen, B. M., Stairs, I. H., \& Thorsett, S. E. 2003, Science, 301, 193

Stappers, B. W., Bailes, M., Lyne, A. G., et al. 1996, ApJ, 465, L119

Tauris, T. M., Langer, N., \& Kramer, M. 2012, MNRAS, 425, 1601

Verbunt, F. 2003, From X-ray Binaries to Gamma-Ray Bursts: Jan van Paradijs Memorial Symposium, 308, 201

Weisberg, J. M., Nice, D. J., \& Taylor, J. H. 2010, ApJ, 722, 1030

Wijnands, R. \& van der Klis, M. 1998, Nature, 394, 344 